\title{
IMPACTOS ASSOCIADOS À RESOLUÇÃO DOS MODELOS ATMOSFÉRICOS EM MODELOS DE PROGNÓSTICOS DE ONDAS
}

\section{The Impact of the Resolution of Wind Fields on Predictive Wave Models}

\author{
Bárbara Silva Lewis ${ }^{1}$ \\ Izabel Christina Martins Nogueira ${ }^{1}$ \\ Nelson Violante de Carvalho ${ }^{1}$ \\ Wallace Figueiredo de Menezes²

\begin{abstract}
1 Universidade Federal do Rio de Janeiro, Programa de Engenharia Oceânica - PenO/COPPE, Engenharia Oceânica, Rio de Janeiro, RJ, Brasil. barbara@lamma.ufrj.br; izabelcm.nogueira@gmail.com.; violante_carvalho@yahoo.co.uk

2 Universidade Federal do Rio de Janeiro, Departamento de Meteorologia - IGEO/CCMN, Meteorologia, Rio de Janeiro, RJ, Brasil. wallace.menezes@gmail.com
\end{abstract}

\begin{abstract}
:
In this paper the effects of the spatial and temporal resolutions of the wind field on the forecasting of wind waves are discussed. Three different meteorological events that generated waves in the Campos Basin (south-eastern Brazil) were simulated using the mesoscale Brazilian Regional Atmospheric Modeling System (BRAMS) with high spatial resolutions of 20, 60, 100, 300 and 600 $\mathrm{km}$ and temporal resolutions of 1,3 and 6 hours and the results were entered into WAVEWATCH (WW3). Two of these events were associated with the formation of extratropical cyclones in the South Atlantic which propagated with velocities above the local average and with large fetches. In the third event, the waves were associated with small fetches generated by the Subtropical South Atlantic Anticyclone (ASAS). The selection of these meteorological events, each with different physical characteristics, allowed us to evaluate the performance of the wave model as a function of the resolution of the wind field input. Our results indicate that the best spatial resolution in terms of computational cost versus error is $60 \mathrm{~km}$, whereas the different time resolutions impose inconspicuous impact on the wave forecast.
\end{abstract}

Keywords: Meteorological models; Wave modelling; Resolution

\section{Resumo:}

Neste trabalho são avaliados os impactos que as diferentes resoluções temporais e espaciais do campo de vento provocam nos resultados de altura significativa do modelo prognóstico de onda. Com esse objetivo, três eventos meteorológicos que geraram ondulações na Bacia de Campos foram simulados com alta resolução utilizando o modelo de mesoescala Brazilian Regional Atmospheric Modeling System (BRAMS) e os resultados empregados no WAVEWATCH (WW3) 
para as seguintes resoluções espaciais 20,60,100, 300 e $600 \mathrm{~km}$, com resoluções temporais de 1, 3 e 6 h. Dois dos eventos selecionados estavam associados à formação de ciclones no Atlântico Sul que se propagaram com velocidades acima da média e geraram pistas com grande extensão. No terceiro caso, as ondas estavam associadas a pistas de pequenas dimensões espaciais geradas pelo Anticiclone Subtropical do Atlântico Sul (ASAS). A seleção destes casos com diferentes características físicas proporcionou uma melhor avaliação das discrepâncias que as resoluções espaciais e temporais ocasionariam. Tendo em vista os resultados encontrados, sugere-se que, para diminuir os erros, sejam utilizados campos de ventos com espaçamento de até $60 \mathrm{~km}$ e, quanto à resolução temporal, destaca-se que os três intervalos resolveram bem os casos selecionados.

Palavras-chave: Modelagem atmosférica; Modelo de ondas; Resolução computacional

\section{Introdução}

$\mathrm{Na}$ ocorrência de eventos meteorológicos que produzem ventos intensos, ondas excepcionais podem provocar diversos tipos de transtornos às práticas costeiras, como prejuízos econômicos e perdas humanas. Em Innocentini et al. (2003), relata-se algumas avarias sofridas no estado do Rio de Janeiro durante uma ressaca ocorrida em maio de 2001. Este evento foi gerado por um ciclone extratropical que se desenvolveu próximo ao litoral de São Paulo. Durante sua propagação pelo Atlântico, o ciclone provocou ondas tão intensas que, ao atingirem a costa do Rio de Janeiro, deixaram cerca de 160 pessoas desabrigadas e, em Barra de Guaratiba, derrubaram uma muralha de pedra com 25 metros de comprimento.

Uma das maneiras de prever agitação marítima na região costeira é através da modelagem de ondas. Para que as simulações de ondas sejam possíveis são necessários os campos de vento gerados pelos modelos atmosféricos, que são utilizados como dados de entrada ("input"), para os modelos de prognósticos de ondas oceânicas.

Kalnay (2003) apresenta uma interessante discussão sobre modelagem numérica atmosférica e aponta que a melhora na previsão numérica nos últimos 40 anos se deve ao aumento da capacidade de processamento dos computadores, permitindo altas resoluções e aproximações numéricas mais refinadas dos modelos operacionais; a melhora na representação dos processos físicos de pequena escala (nuvens, precipitação, transferências turbulentas de calor, umidade, quantidade de movimento e radiação) nos modelos; o uso de métodos mais acurados de assimilação de dados, que resultam em condições iniciais mais adequadas para os modelos gerarem os melhores resultados; e o aumento da disponibilidade de dados, especialmente dados de satélite e aviões sobre os oceanos e Hemisfério Sul.

Este avanço na capacidade de processamento dos computadores permitiu a confecção de experimentos com altíssimas resoluções e, com isso, resultados com maior detalhamento atmosférico. Entretanto, essas vantagens levantaram alguns questionamentos em relação até onde uma elevada resolução espacial e temporal pode apresentar ganhos nos resultados, quando comparados com dados observados.

$\mathrm{Na}$ modelagem atmosférica alguns autores compararam os resultados de simulações com diferentes resoluções espaciais e dados observados, dentre eles destacam-se Zhong et al. (2003), Mass et al. (2002) e Rife et al. (2005). Todos os autores concluíram que o aumento da resolução espacial apresentou resultados com erros menores. Mass et al. (2002) completa ainda que o ganho 
ocorreu ao diminuir o espaçamento de grade de $32 \mathrm{~km}$ para $12 \mathrm{~km}$, mas que ao aumentar resolução de $12 \mathrm{~km}$ para $4 \mathrm{~km}$ não observou vantagens, sugerindo que a resolução ideal do modelo atmosférico para a região analisada fosse entre 10 e $15 \mathrm{~km}$.

Uma possível fonte de erros dos modelos de geração e propagação de onda está associada aos campos de ventos utilizados como input dos modelos de ondas. Isto porque, como dito anteriormente, existem erros numéricos associados também aos modelos atmosféricos, ressaltando-se o fato dos modelos não conseguirem reproduzir completamente a enorme diversidade de fenômenos que influenciam a evolução das condições atmosféricas e a dependência da boa qualidade dos dados observados.

Ostritz et al. (2012), ao conferir as melhorias que os ventos podem gerar nos resultados dos modelos de onda, percebeu-se que alta resolução espacial e temporal gera impactos positivos na estimativa das ondas, principalmente em relação à altura significativa.

O ganho nos resultados numéricos de ondas ao aumentar a resolução espacial dos modelos atmosféricos foi amplamente discutido por Cavaleri e Bertotti (2003) e Cavaleri e Bertotti (2006). Em Cavaleri e Bertotti (2006), foram testadas diferentes configurações dos modelos, inicialmente o espaçamento da grade de $188 \mathrm{~km}$ foi reduzido para $31 \mathrm{~km}$ e posteriormente para $25 \mathrm{~km}$. Com isso, os autores concluíram que o aumento de resolução aproxima, de maneira geral, os resultados aos dados medidos, diminuindo os valores de viés, ou seja, a parcela sistemática do erro.

Nos trabalhos de Palmeira et al. (2012) e Ostritz et al. (2012) o modelo WAVEWATCHIII é utilizado com a finalidade de avaliar a importância do detalhamento dos ventos. Em ambos, foram comparados resultados dos modelos de ondas para simulações com ventos com diferentes resoluções espaciais de distintos modelos atmosféricos, sendo o campo de vento mais resoluto obtido através de modelos de mesoescala. Os autores concluíram que os modelos de mesoescala proporcionaram melhores resultados que os globais (com espaçamento de grade maior), apontando que a prática de regionalização dinâmica, ou seja, diminuir o espaçamento de grade através da modelagem é valido. Os autores ressaltaram ainda que o aumento da resolução espacial teve uma maior importância que o aumento da resolução temporal.

Embora alguns autores tenham desenvolvido pesquisas relacionadas aos erros encontrados na integração numérica dos modelos de ondas, ou os impactos que as resoluções do campo de ventos apresentam nos resultados, ainda há poucas pesquisas que apresentem quais as resoluções espaciais e temporais são ideais para representar as condições de mar da costa Brasileira. No presente trabalho, os impactos destas diferentes resoluções espaciais e temporais serão testados para uma área limitada, buscando quais configurações de resolução espacial e temporal geram resultados mais representativos dos estados de mar da região sudeste do Brasil.

\section{Material e Métodos}

A região escolhida para o estudo foi a Bacia de Campos, área de grande importância econômica para o país. Esta está localizada entre o litoral norte do Estado do Rio de Janeiro e o sul do Estado do Espírito Santo (Figura 1- caixa vermelha), limitada a norte pelo Arco de Vitória e, ao sul pelo Arco de Cabo Frio. Ao longo de mais de três décadas de exploração petrolífera, mais de 1.600 poços foram perfurados, sendo que, economicamente, é a bacia brasileira mais prolífica, concentrando mais de 90\% das reservas petrolíferas brasileiras atuais (Winter et al. (2007)). 
Das características meteo-oceanográficas da área de estudo, destaca-se que, os principais sistemas meteorológicos que determinam o clima de ondas no Atlântico Sul e também na Bacia de Campos são os ciclones extratropicais, os anticiclones migratórios e o Anticiclone Subtropical do Atlântico Sul. Os ventos associados ao ASAS geram ondas que variam de leste a norte em direção à costa do Rio de Janeiro, enquanto que os anticiclones e ciclones migratórios estão associados às ondulações geradas a longas distâncias no Atlântico e se propagam até a costa com direções que variam entre SE e SW.

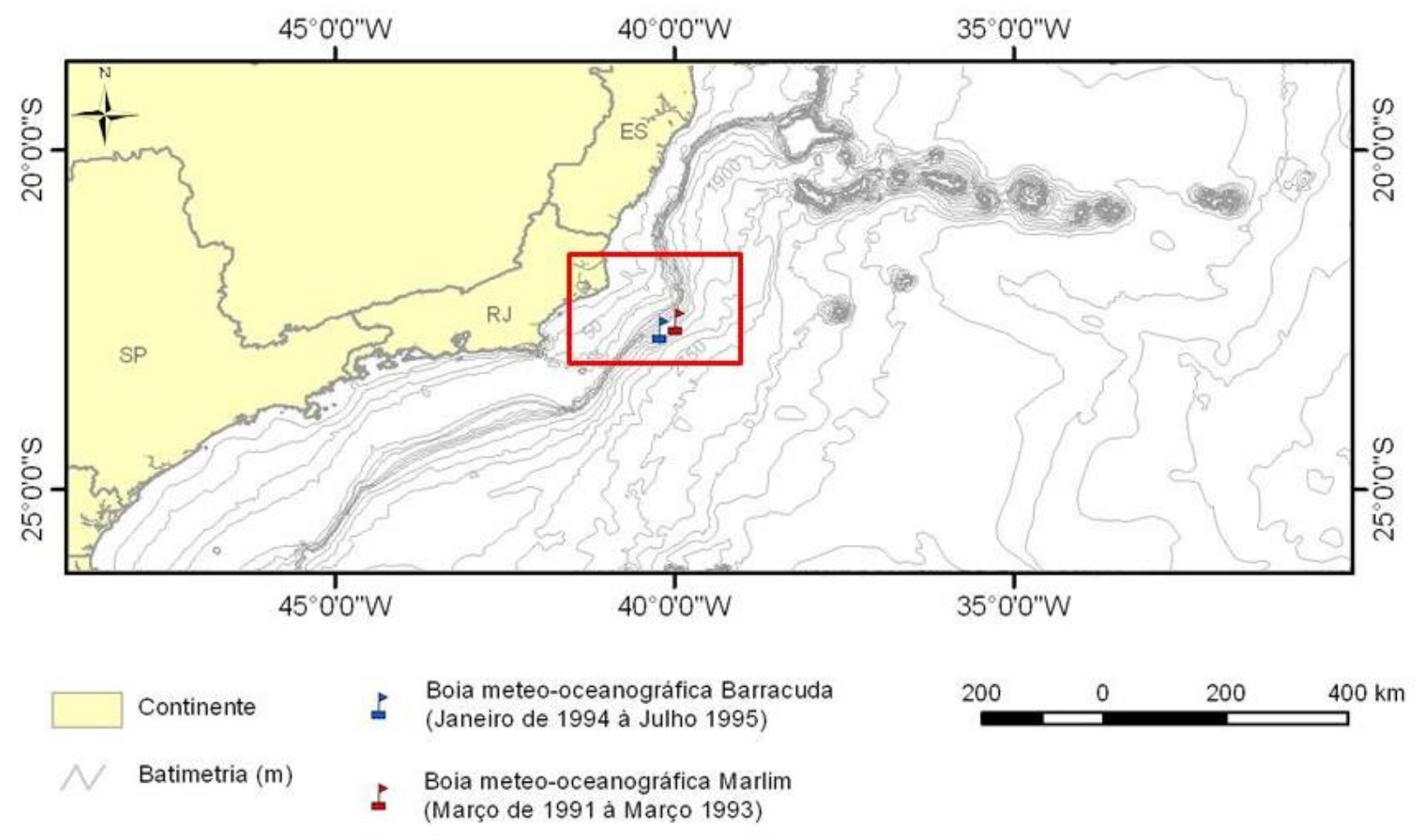

Figura 1: Região da Bacia de Campos e localização da boia em Marlim e em Barracuda.

Foram realizadas simulações com modelo atmosférico de mesoescala BRAMS para obtenção do campo de vento para eventos meteorológicos interessantes e que geraram ondas com grandes alturas significativas na região analisada. A altura significativa das ondas, Hs, é a média do terço das maiores ondas de um registro, no domínio do tempo, e é o parâmetro mais comumente usado para definir o grau de severidade do mar.

Estes campos de vento foram implementados no modelo prognóstico de ondas WAVEWATCH III e os resultados de Hs comparados com dados da boia Marlim-Barracuda fundeada na área de estudo.

A boia meteo-oceanográfica do tipo heave-pitch-roll foi fundeada nas coordenadas $22^{\circ} 3^{\prime} \mathrm{S}$ e 39 $58^{\prime}$ 'W (Campo de Marlin), a uma profundidade de 1250 metros, durante o período de março de 1991 a março de 1993. Entre janeiro de 1994 e julho de 1995 esta boia foi reinstalada no Campo de Barracuda, na posição $22^{\circ} 38^{\prime} \mathrm{S}$ e $40^{\circ} 12^{\prime} \mathrm{W}$, e a uma profundidade de 1050 metros (PINHO, 2003).

Para verificar o comportamento das ondas e do vento nos meses escolhidos foi utilizada a Plotting the Evolution of the Directional Spectrum- PLEDS. A PLEDS é um complemento da técnica DAAT, que é um acrônimo para Directional Analysis with Adaptive Techniques desenvolvida por Parente (1999). A DAAT é uma técnica do tipo tempo-frequência para análise direcional de ondas e permite detectar, para uma mesma faixa de frequência, a ocorrência de ondas de diversas 
direções, aumentando de forma significativa à resolução direcional (Vale (2012)). Empregando a PLEDS é possível observar a propagação das ondas por faixas de frequências, a energia da onda e a direção da mesma ao longo do tempo.

Na PLEDS (Figura 2) as elevações curvas no gráfico são proporcionais à energia dos espectros de ondas (1), para cada faixa de períodos, sendo a escala de cores que representa a faixa de períodos (2) está demonstrada na legenda da PLEDS. A evolução das ondas no tempo pode ser observada no eixo das ordenadas, que indicam os dias (3). O eixo das abscissas indica a direção em graus do vento e da onda (4). As barras verticais representam a velocidade do vento (5), sendo que cinco divisões horizontais correspondem a aproximadamente $10 \mathrm{~ms}^{-1}$ e a escala de cores da velocidade do vento também é apresentada na legenda (6) da PLEDS.

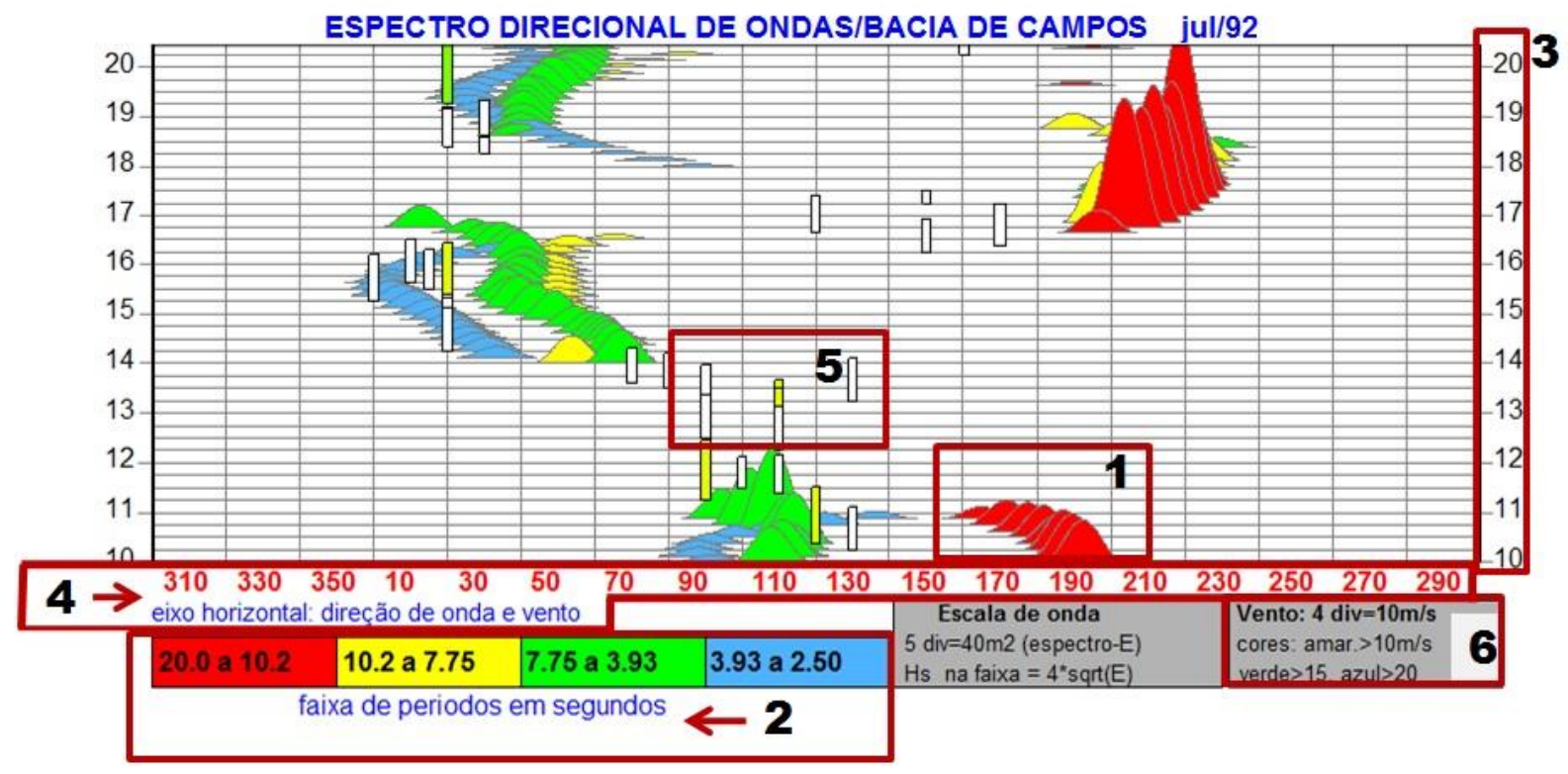

Figura 2: Exemplo da evolução do espectro direcional utilizando a PLEDS desenvolvida por PARENTE (1999).

\subsection{Seleção de Casos}

Neste trabalho foram selecionados três fenômenos meteorológicos responsáveis pela geração de ondas que afetaram a agitação marítima da Bacia de Campos. Estes fenômenos apresentaram características peculiares e foram escolhidos para que as possíveis deficiências do modelo de ondas, em relação às resoluções temporais e espaciais, pudessem ser observadas. Com a escolha desses casos em particular, espera-se observar que, os fenômenos atmosféricos que possuem pequenas dimensões espaciais e se desloquem com altas velocidades não sejam bem resolvidos pelo modelo de ondas quando este é implementado com espaçamentos de grades e resoluções temporais pouco refinadas.

Alguns autores adaptaram o método automático de análise da trajetória de centros de baixa e alta pressão proposto por Murray e Simmonds (1991) para estimar os valores de velocidade de deslocamento médio dos ciclones. Dentre eles Pesquero (2001) aferiu 23 e $26 \mathrm{~km} / \mathrm{h}$ utilizando dados dos modelos COLA e T062L28, Mendes (2006) e Mendes et al.(2009) o valor de $21 \mathrm{~km} / \mathrm{h}$ 
empregando as reanálises do NCEP e Reboita (2008) estimou $29 \mathrm{~km} / \mathrm{h}$ com dados do Modelo RegCM.

Dessa forma, foi considerado um ciclone com alta velocidade de deslocamento aquele em que os valores da velocidade de deslocamento fossem superiores aos maiores valores encontrados na literatura citada.

\subsubsection{Caso de Março de 1992}

As ondulações registradas pela boia Marlim entre os dias 24 e 26 de março de 1992 foram analisadas por Silva (2008). O evento foi classificado como Bom Tempo com ondulação (Seixas (1997), Violante Carvalho (1998) e Parente (1999)) e a ocorrência de ondas dispersivas na Bacia de Campos foi associada a um ciclone gerado no extremo sul da América do Sul no dia 21. O ciclone observado gerou uma pista com grande extensão sobre o Atlântico. Esta pista, teoricamente, poderia ser mais facilmente resolvida por grades com resoluções mais grosseiras no modelo de onda. Já a velocidade de deslocamento deste centro de baixa pressão foi de $33 \mathrm{~km} / \mathrm{h}$, sendo este valor um pouco acima da média de deslocamento apresentada anteriormente, e isto poderia apresentar algumas discrepâncias entre as diferentes resoluções temporais empregadas na modelagem de ondas. Ressalta-se que este valor foi estimado de forma subjetiva através da análise dos campos de pressão atmosférica no domínio do tempo.

A Figura 3 ilustra a PLEDS para o período do dia 22 a 31 de março. Pode ser observada a persistência de ondas na faixa do mar local com direções entre NE e E, possivelmente devido à influência do ASAS. Mesmo este padrão sendo mantido por tantos dias, também foi possível observar a chegada de ondulação com direção Sul, girando para Sudeste, entre o dia 22 e 23. O evento mais energético do mês de março ocorreu nos dias 24 e 25, quando ondas se propagaram para a Bacia de Campos com direção SW e períodos maiores que 10 segundos.

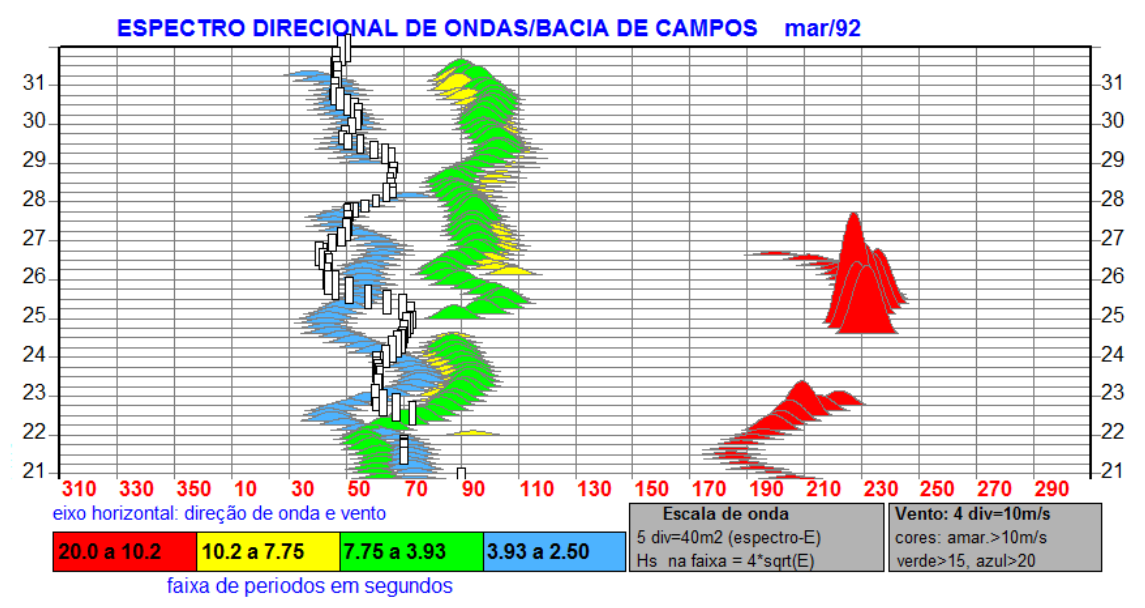

Figura 3: PLEDS entre os dias 22 e 31 de março de 1992. 


\subsubsection{Caso de Agosto de 1992}

O ciclone responsável pela formação das ondulações de sudoeste (SW) e de maior pico energético observadas na Bacia de Campos no dia 25 de agosto de 1992 alcançou uma velocidade de média de deslocamento em $24 \mathrm{~h}$ de aproximadamente $35 \mathrm{~km} / \mathrm{h}$. Esta velocidade, assim como em março, também foi um pouco superior a média encontrada. Este ciclone teve uma considerável mudança de dimensão espacial, sendo de proporções pequenas na formação e atingindo rapidamente uma grande dimensão espacial sobre o Atlântico nas primeiras 24 horas. Esta rápida mudança nas feições espaciais do ciclone, em um período curto, poderia resultar em discrepâncias ao tratar de diferentes resoluções, principalmente resoluções temporais.

Observando a PLEDS para o mês de agosto (Figura 4) não foi constatado um padrão de ondas de NE e E, como visto em março, isto pode significar a pouca influência de pistas com estas direções geradas pelo ASAS. Entre os dias 22 e 24, foram observadas ondas com direções entre SE e S, com pouca energia e períodos entre 8 e 10 segundos. Esta situação foi interrompida com a chegada de ondulações com períodos maiores que 10 s e direção de SW.

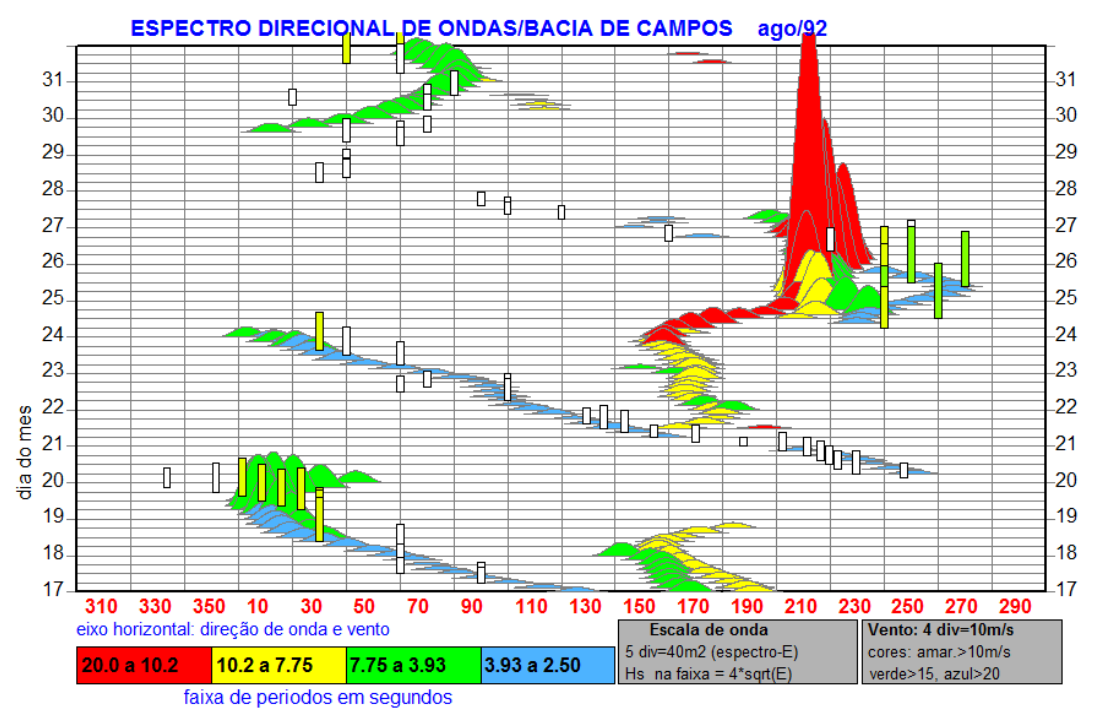

Figura 4: PLEDS entre os dias 17 e 31 de agosto de 1992.

\subsubsection{Caso de Dezembro de 1992}

Este caso foi selecionado devido a dois fatores: i) Por ser tratar de um evento onde a formação de ondas estava associada ao ASAS. Neste caso, o objetivo era analisar um novo perfil de ondas, de forma que, nesta nova escolha, as ondas de maior energia estivessem associadas a um mar local, com direções entre E e N. Nos casos anteriormente analisados, as ondas de maior energia estavam associadas a formação de ciclone no Atlântico Sul e a chegada do marulho com direção SW. Vale (2012) associou as ondas observadas no dia 11 e 12 de dezembro (Figura 5) à formação de um ciclone bem próximo da costa do Rio de Janeiro, o qual provocou um aumento no gradiente de pressão entre este centro de baixa pressão e a circulação do Anticiclone Subtropical do Atlântico 
Sul, intensificando os ventos de Nordeste; ii) a escolha do caso de dezembro também foi em virtude do fato de que a pista de geração associada ao ASAS apresentou uma pequena extensão, podendo ser interessante para explorar as capacidades do modelo dentro das diferentes resoluções espaciais.

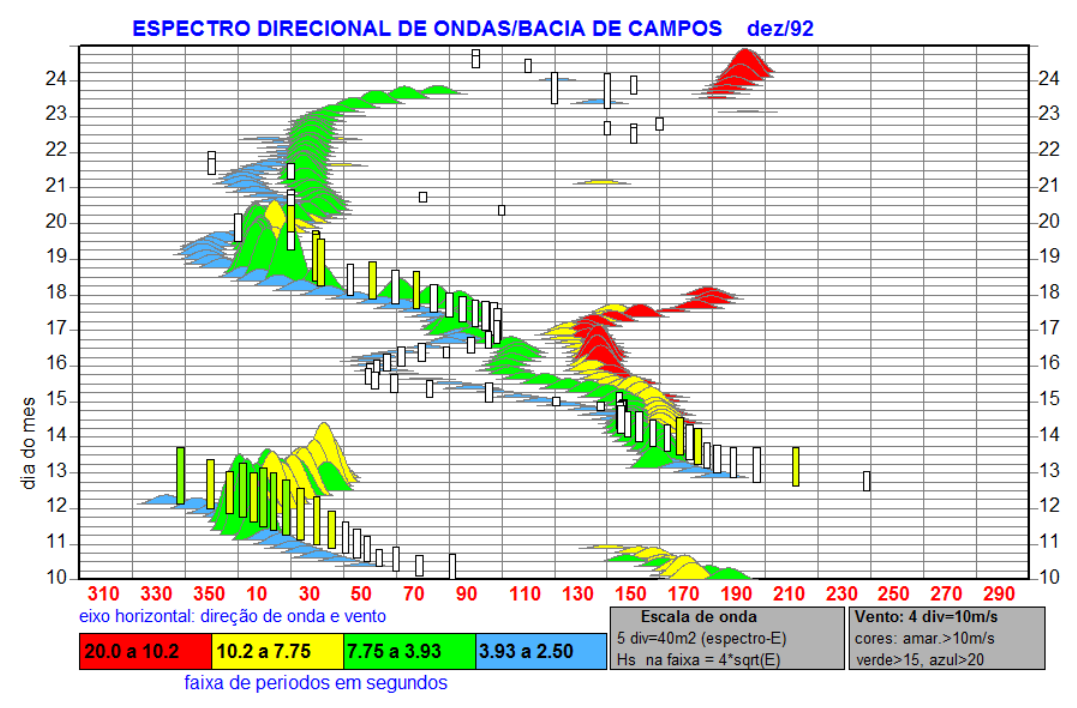

Figura 5: PLEDS entre os dias 10 a 24 de dezembro de 1992.

\subsection{Modelagem Atmosférica}

A modelagem atmosférica foi realizada através do BRAMS, versão 4.2, que é um modelo de previsão numérica desenvolvido com objetivo de simular as circulações atmosféricas com diferentes escalas, variando desde a microescala até simulação de grandes vórtices (LES- em inglês Large Eddy Simulations) da Camada Limite Planetária (Freitas et al. (2009)). O BRAMS é resultado de um projeto de pesquisa financiado pela FINEP em 2002/2003 com objetivo de produzir uma versão do modelo de mesoescala RAMS (Regional Atmospheric Modeling System) (Pielke et al. (1991); Tripoli e Cotton (1982); Tremback e Kessler (1985)) adaptado para os trópicos.

As condições iniciais e de contorno do modelo necessárias para implantação do modelo de mesoescala BRAMS foram provenientes das reanálises do Climate Forecast System Reanalysis (CFSR - http://rda.ucar.edu/pub/cfsr.html), com resolução espacial de $0,5^{\circ}$ para todo globo e resolução temporal de 6 horas.

Com o BRAMS foram realizadas integrações para o caso de março do dia 15 de março 1992 às $00 Z$ até dia 31 de março 1992 às 00Z, do caso de agosto do dia 15 de agosto de 1992 às $00 Z$ até dia 27 de agosto de 1992 às $12 Z$ e para o caso de dezembro do dia 01 de dezembro de 1992 às $00 Z$ até dia 31 de dezembro 1992 às 00Z.

O número de pontos de grade da simulação inicial foi de 230 na horizontal e 365 na vertical, com resolução espacial de $20 \mathrm{~km}$ e temporal de 1 hora, sendo o centro da grade em $-36 \mathrm{~S}$ e $-44 \mathrm{~W}$.

A partir dos resultados do modelo de atmosférico BRAMS para a resolução de $20 \mathrm{~km}$ em $1 \mathrm{~h}$, foi realizado um processo para diminuir o número de pontos da grade numérica e com isso aumentar 
o espaçamento da grade no tempo e no espaço. Assim, foram gerados novos campos de ventos com a configuração apresentada na Tabela 1. Este processo de gerar novas configurações do campo de vento foi realizado através de um algoritmo aplicado computacionalmente que descartava os valores do vento para pontos de grade que não apresentavam a resolução escolhida para o experimento.

Tabela 1: Informações das Simulações: resoluções espaciais e números de pontos

\begin{tabular}{c|c|c|c|c|c} 
Resolução & $\mathbf{2 0} \mathbf{~ k m}$ & $\mathbf{6 0} \mathbf{~ k m}$ & $\mathbf{1 0 0} \mathbf{~ k m}$ & $\mathbf{3 0 0} \mathbf{~ k m}$ & $\mathbf{6 0 0} \mathbf{~ k m}$ \\
\hline Número de pontos em X & 230 & 77 & 46 & 16 & 8 \\
\hline Número de pontos em Y & 365 & 122 & 73 & 25 & 13
\end{tabular}

A Figura 6 ilustra uma representação do processo para diminuir a resolução espacial. Este método foi realizado para garantir a utilização do mesmo campo de vento, indiferente da grade empregada, modificando assim apenas a resolução. Ao utilizar o mesmo campo de vento, apenas modificando os espaçamentos de grades, é descartada a possibilidade de que as diferenças encontradas no campo de ondas fossem inerentes aos distintos métodos físicos e matemáticos dos modelos atmosféricos.

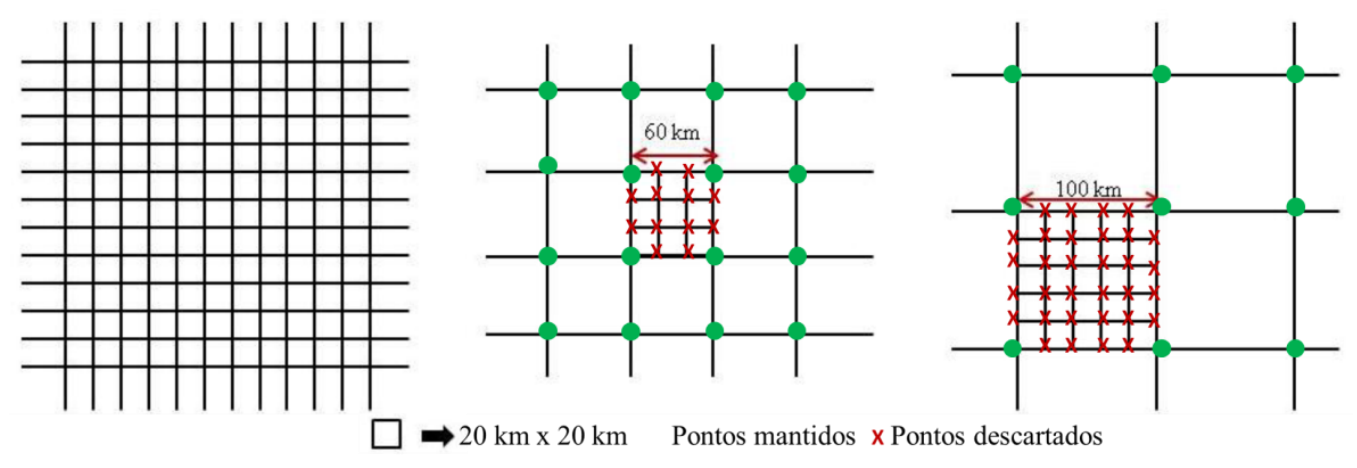

Figura 6: Exemplo do método para diminuir a resolução espacial da grade

\subsection{Modelagem de Ondas}

Para modelagem de ondas a partir dos campos de ventos apresentados na TABELA 1 foi utilizado o WAVEWATCH (WW3), versão 3.14, que é um modelo de geração e propagação de ondas de terceira geração para águas intermediárias e profundas, baseado no trabalho realizado pelo WAM group e desenvolvido pela National Center for Environmental Prediction (NCEP). O modelo resolve a equação de balanço do espectro de energia em função do número de onda e da direção. A equação do balanço de energia representa a variação do estado do mar causado por processos adiabáticos, como advecção, e pelas funções físicas da fonte de geração de ondas do mar pelo vento, dissipação devido à quebra da crista das ondas e a interações não lineares (Bidlot (2007) apud Tolman (2009)). A parametrização dos termos de ganho e dissipação de energia, bem como a representação das interações não lineares foram propostas por Tolman e Chalikov (1996) apud Tolman (2009) e a Discrete Interaction Approximation (Hasselmann et al. (1985) apud Tolman (2009)), respectivamente. 
Os resultados de Hs das simulações de ondas para estas novas 15 configurações do campo de ventos (5 resoluções espaciais em 3 diferentes resoluções temporais) foram comparados com os dados da boia meteo-oceanográfica Marlin-Barracuda situada na Bacia de Campos e analisados por faixas de períodos. Desta maneira, as integrações do espectro (equação 1) para o cálculo de Hs (equação 2) foram realizadas para as seguintes faixas de períodos: Faixa A para períodos acima de 10 s, Faixa B de 8 até 10 s, Faixa C de 6 até 8 s, Faixa D de 4 até 6 s, Faixa E de 2 até 4 s.

$$
\begin{gathered}
E(f)=\int_{0}^{\infty} S(f) d f=m_{0} \\
H_{s}=4,01 \sqrt{m_{0}}
\end{gathered}
$$

Onde $\mathrm{S}(\mathrm{f})$ é o espectro de frequência da série de elevações da superfície livre da água e mo é a área total (ou energia total) do espectro de ondas.

Um exemplo de como seria a aplicação das equações 1 e 2 para a Faixa C, com período compreendido entre 6 e 8 s, seria: $E(f)=\int_{6}^{8} S(f) d f=m_{c}$ e $H_{c}=4,01 \sqrt{m_{c}}$.

\section{Resultados e Discussão}

Ao analisar os resultados por faixas de período foi possível observar que houve pouca diferença nos valores de altura significativa das simulações de 1, 3 e 6 horas. Por esta razão, nas próximas seções serão apresentados apenas os resultados para simulação de 1 hora.

Na tabela 2 são expostos os resultados do Viés, do Erro Médio Quadrático (EMQ) e do Coeficiente de Correlação (CF.CORR) para as simulações de $20 \mathrm{~km}$ em 1, 3 e 6 horas dos casos de março, agosto e dezembro de 1992.

Tabela 2: Parâmetros estatísticos de Altura Significativa

\begin{tabular}{c|c|c|c|c|c|c|c|c|c}
\cline { 2 - 9 } & \multicolumn{3}{c|}{ Março - HS } & \multicolumn{3}{c|}{ Agosto - HS } & \multicolumn{3}{c}{ Dezembro - HS } \\
\cline { 2 - 9 } \multicolumn{1}{c|}{} & VIÉS & EMQ & CF. CORR & VIÉS & EMQ & CF. CORR & VIÉS & EMQ & CF. CORR \\
\hline $1 \mathrm{~h}$ & -0.5060 & 0.6670 & 0.6250 & -0.3650 & 0.7330 & 0.9310 & -0.3700 & 0.4770 & 0.8490 \\
\hline $3 \mathrm{~h}$ & -0.5100 & 0.6690 & 0.6260 & -0.3770 & 0.7440 & 0.9300 & -0.3760 & 0.4820 & 0.8480 \\
\hline $6 \mathrm{~h}$ & -0.5190 & 0.6760 & 0.6280 & -0.4110 & 0.7740 & 0.9260 & -0.3940 & 0.4980 & 0.8450 \\
\hline
\end{tabular}

De uma maneira geral, para todos os casos, as simulações subestimaram os dados da boia, sendo o caso de março o que apresentou maior valor de viés e menor coeficiente de correlação. O caso de agosto foi o que apresentou melhor correlação, com valores maiores que 0,92 . Os menores valores de EMQ foram observados no caso de dezembro, isto pode indicar uma maior precisão do modelo de ondas para este caso. Embora os valores de Hs tenham sido subestimados para grande 
parte dos dados, observa-se que o coeficiente de correlação foi alto para os três casos, deixando claro que o modelo conseguiu simular adequadamente a tendência dos dados registrados pela boia.

\subsection{Resultados do Caso de Março}

Analisando o viés para Hs por faixa de período (Figura 7.a), observa-se que entre 2 e 4 segundos as simulações de 20, 60 e $100 \mathrm{~km}$ superestimaram os dados observados, sendo que a partir de $4 \mathrm{~s}$, todas as simulações foram subestimadas, exceto a de $100 \mathrm{~km}$, que entre 4 e 6 s superestimou os observados. Os valores de viés, para as resoluções de 20, 60 e $100 \mathrm{~km}$ foram mais baixos (menores que $0,05 \mathrm{~m}$ ) na faixa entre 2 e 6 segundos e valores maiores que $0,32 \mathrm{~m}$ para as faixas de período de acima de $6 \mathrm{~s}$ (Faixas $\mathrm{C}$, B e A). Isto pode representar que os resultados das simulações de ondas associadas ao mar local, estado do mar este que persistiu na Bacia de Campos para todo período analisado (Figura 3), apresentaram valores de Hs mais próximos do registro da boia do que ondas com períodos maiores que 6 segundos.

Na Figura 7.b observa-se que os menores valores para o EMQ de Hs estão associados as ondas de alta frequência (menores períodos), sendo que o maior valor de EMQ foi de 0,68 m encontrado na simulação de $600 \mathrm{~km}$ em 1 horas. Neste gráfico fica evidente que os maiores erros estão associados a ondas com períodos acima de 10 segundos que, para este evento de março, estavam relacionados com a chegada de ondulações geradas em baixas latitudes.

As simulações de $20 \mathrm{~km}, 60 \mathrm{~km}$ e $100 \mathrm{~km}$ apresentaram concordância para todas as faixas de períodos. As diferenças entre as rodadas de pior resolução $(600 \mathrm{~km})$ e de melhor resolução $(20$ $\mathrm{km}$ ) ficaram mais evidentes entre 4 e $6 \mathrm{~s}, 6$ e $8 \mathrm{~s}$ e os períodos acima de $10 \mathrm{~s}$ com valores de 0,17 $\mathrm{m}, 0,15 \mathrm{~m}$ e $0,11 \mathrm{~m}$, respectivamente.

No caso de março as ondulações observadas acima de 8 segundos, de acordo com a PLEDS (Figura 3), estavam associadas principalmente ao ciclone formado no sul do Atlântico e com ondas geradas pelo ASAS, com direção E. Para estas faixas (acima de $8 \mathrm{~s}$ ), ocorreu certa concordância dos valores de EMQ da Hs entre as simulações até $300 \mathrm{~km}$ (Figura 7.b) . No geral, isto pode significar que neste caso de março, tanto o ciclone responsável pelas ondulações de SW quanto o ASAS foram bem representados por resoluções menos refinadas, como a de 100 e até mesmo $300 \mathrm{~km}$. 


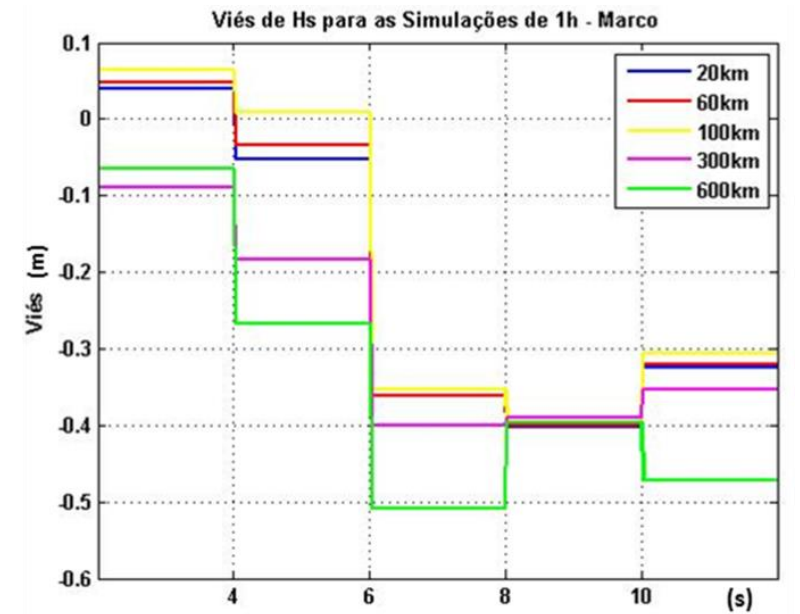

(a)

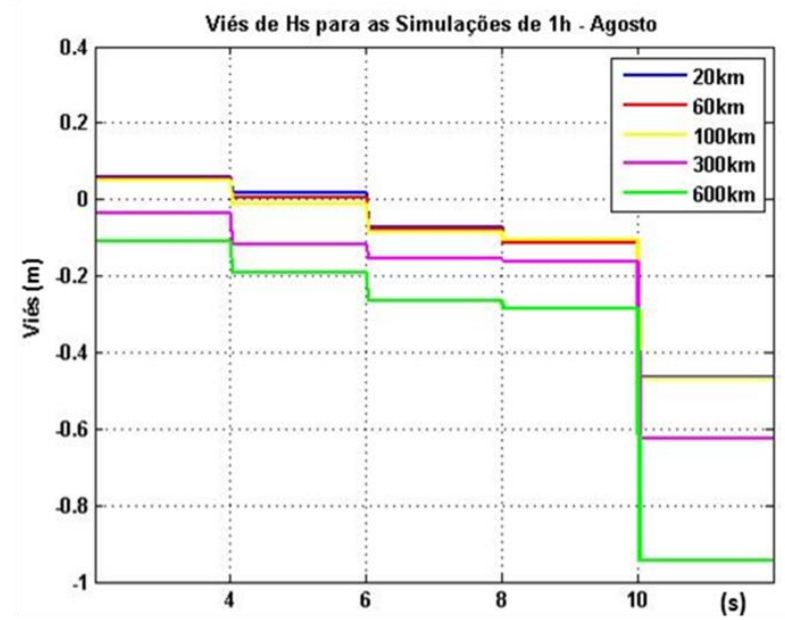

(c)

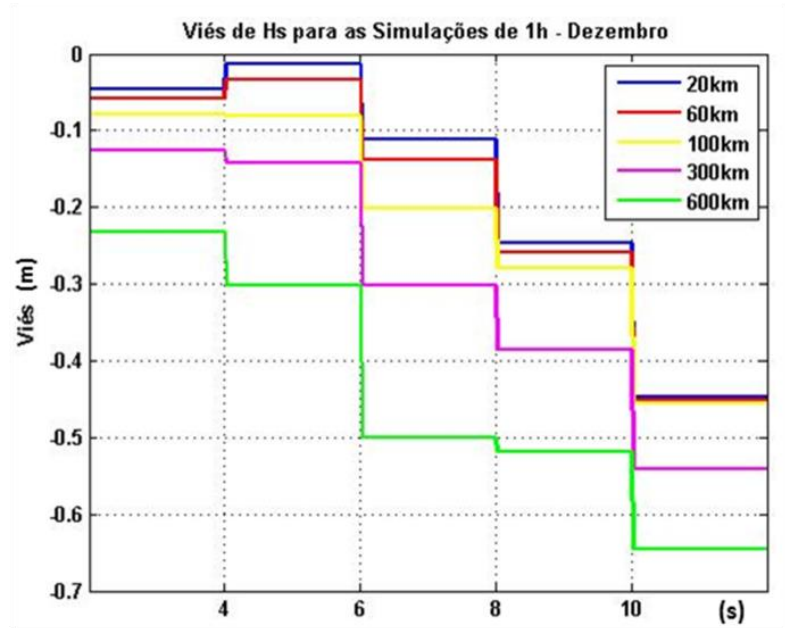

(e)

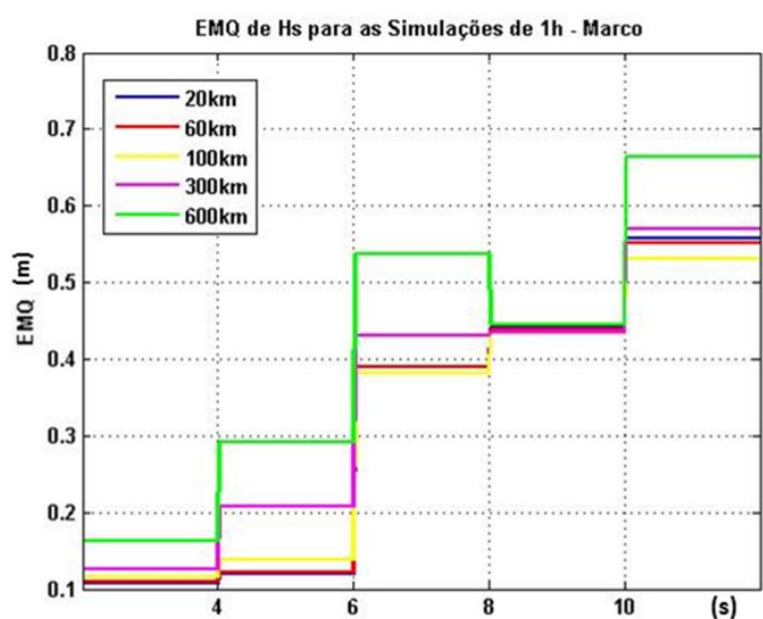

(b)

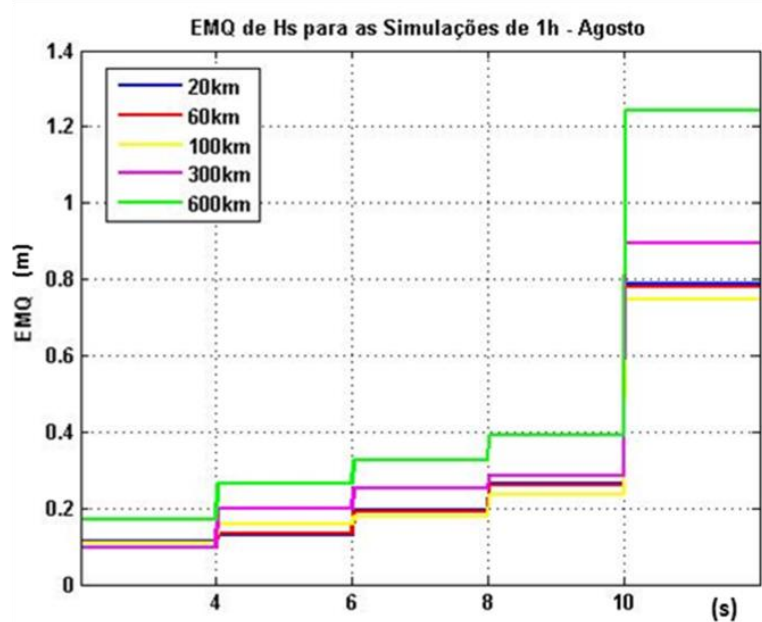

(d)

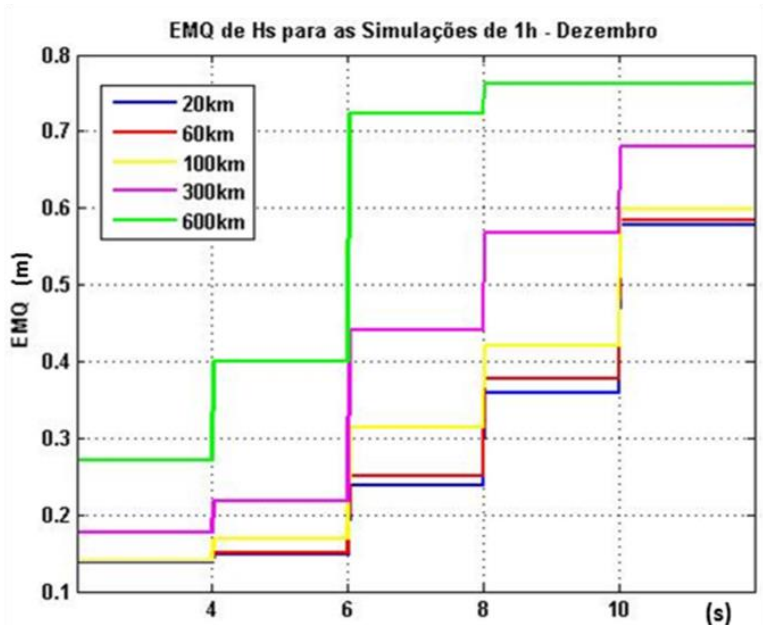

(f)

Figura 7: (a), (c) e (e) Viés e (b), (d) e (f). EMQ da altura significativa por faixas de períodos para os resultados das simulações do WW3 dos casos de março, agosto e dezembro utilizando como entrada as resoluções espaciais de 20,60, 100, 300 e $600 \mathrm{~km}$ e temporal de 1 hora. 


\subsection{Resultados do Caso de Agosto}

Analisando o viés por faixa de período para o parâmetro de Hs (Figura 7.c), observa-se que, para as rodadas de 20,60 e $100 \mathrm{~km}$, o valor absoluto do viés é maior para maiores períodos. Para faixas acima de $6 \mathrm{~s}$ segundos todas as simulações subestimaram os observados. As simulações de $300 \mathrm{e}$ $600 \mathrm{~km}$ apresentaram resultados subestimados para todas as faixas de períodos quando comparadas a boia, enquanto que as rodadas de 20,60 e $100 \mathrm{~km}$ os resultados foram levemente superiores aos dados da boia nas faixas entre 2 e 6 segundos.

De uma maneira geral, valores de viés, para todas as simulações, apresentaram uma boa concordância entre si, com diferenças maiores apenas para faixa A (acima de $10 \mathrm{~s}$ ). Ressalta-se também que os erros foram menores para as faixas de período até $10 \mathrm{~s}$ (Faixas E, D, C e B).

Para os valores de EMQ da altura significativa no caso de agosto fica evidente como os erros do modelo de ondas aumentam para maiores períodos. As simulações de 20, 60 e $100 \mathrm{~km}$ apresentaram boa concordância (Figura 7.d), sendo a rodada que apresentou maiores valores de EMQ foi a de $600 \mathrm{~km}$. A faixa de período com maior EMQ foi acima de $10 \mathrm{~s}$, com valor de 1,24 $\mathrm{m}$, enquanto que faixas entre os períodos de 2 a $10 \mathrm{~s}$ (faixas E, D, C e B) apresentaram erros menores. A simulação de $100 \mathrm{~km}$ apresentou valores de EMQ sutilmente menores que as simulações de $20 \mathrm{~km}$ e $60 \mathrm{~km}$ para períodos acima de $6 \mathrm{~s}$.

No caso de agosto ficou evidente o aumento dos valores de EMQ para ondas com períodos acima de 10 segundos. Estas ondas atingiram a Bacia de Campos com grande energia (Figura 4) e estavam associadas a uma pista de SW gerada por um ciclone. Neste caso, a energia associada ao marulho era alta, o que sugere uma possível deficiência do modelo de ondas em simular eventos mais energéticos.

Os casos de março e de agosto mostraram certa similaridade, pois em ambos os casos, a simulação de $100 \mathrm{~km}$ apresentou reduzidos valores de EMQ para períodos acima de 6 segundos.

Assim como no caso de março, o caso de agosto foi bem representado pelas resoluções espaciais mais baixas, como de 100, 300 e incluindo a de $600 \mathrm{~km}$ até $10 \mathrm{~s}$, sendo que em Março a resolução de $600 \mathrm{~km}$ apresentou mais discordância em outras faixas de período.

Pelo gráfico de EMQ para Hs (Figura 7.d) fica evidente que o valor mais alto de EMQ está na representação do marulho, períodos maiores de $10 \mathrm{~s}$, sendo que o valor de EMQ relacionado ao marulho foi maior que o dobro do observado nas faixas de entre 2 e 10 segundos.

Esta boa representação das resoluções até $300 \mathrm{~km}$ mostra que os ciclones e anticiclones associados à geração de ondas evidenciada na PLEDS de março e agosto (Figura 3 e Figura 4) também podem ser bem resolvidos por baixas resoluções espaciais, mas que ainda existe uma maior dificuldade em resolver os sistemas associados ao marulho mesmo com simulações com resoluções mais refinadas.

\subsection{Resultados do Caso de Dezembro}

Para o viés de Hs (Figura 7.e), todas as simulações subestimaram os dados das boias para todas as faixas de períodos, percebe-se ainda que o valor absoluto de viés aumenta para maiores períodos.

Nas simulações do mês de dezembro ficaram mais evidentes as diferenças de altura significativa entre as simulações de maiores resoluções $(20$ e 60 km) e de menores resoluções $(100,300$ e 600 
$\mathrm{km}$ ). As piores resoluções apresentaram maiores valores de EMQ (Figura 7.f), sendo que o maior valor observado foi de $0,77 \mathrm{~m}$ na rodada $600 \mathrm{~km}$ para períodos maiores que $8 \mathrm{~s}$. Assim como nos outros casos analisados, o maior valor de EMQ foi observado nas ondas com períodos acima de 10 s (Faixa A).

Observando novamente a PLEDS de dezembro (Figura 5), percebe-se que as ondas que continham mais energia registradas na Bacia de Campos tinham períodos entre 4 e $10 \mathrm{~s}$ (faixas $\mathrm{D}, \mathrm{C}$ e B). Estas estavam associadas principalmente a intensificação do ASAS gerando uma pista com direções entre NE e N, como discutido na seção 2.2 de seleção de casos.

No caso de dezembro, a configuração do ASAS foi responsável pela formação de uma pista de Nordeste próxima a Bacia de Campos e, consequentemente, a geração de ondas em frequências mais altas, características de mar local (Figura 5). No caso de agosto, que foi caracterizado principalmente pela passagem de um ciclone, o erro nas faixas de períodos entre 6 e 10 segundos foram menores do que no caso de dezembro. Isto sugere que os eventos de mar local observados em dezembro possivelmente foram mal interpretados pelos modelos.

Em dezembro, observa-se também que a resolução de $100 \mathrm{~km}$ não apresentou bons resultados para períodos acima de 6 segundos, como tinha sido visto nos casos de março e agosto. Neste caso, foram obtidos erros maiores que as resoluções mais refinadas de 20 e $60 \mathrm{~km}$. Isto pode estar associado à diferença dos sistemas que ditaram as pistas destes diferentes eventos. Enquanto que nos casos de março e agosto os eventos mais energéticos das ondulações estavam associados a pistas com grandes extensões espaciais, que poderiam ser facilmente interpretadas pela simulação de $100 \mathrm{~km}$, no caso de dezembro as pistas tinham dimensões relativamente pequenas, o que pode ter gerado uma má interpretação por parte do modelo de ondas devido à utilização de resoluções de vento pouco refinadas $(100 \mathrm{~km}, 300 \mathrm{~km}$ e $600 \mathrm{~km})$.

\section{Considerações Finais}

Destaca-se que não houve muita diferença nos valores encontrados entre as simulações de 1 hora, 3 horas e de 6 horas, isso pode significar que a interpolação de vento realizada pelo WW3 para 1 hora não provoca perda de informação, ou seja, representa bem o vento. Além disso, sugere-se que a utilização de ventos com resolução temporal mais grosseira como 3 e 6 horas também são suficientes para simular as ondulações geradas no Atlântico Sul, uma vez que, dentre os eventos analisados, a velocidade média de deslocamento máximo do ciclone foi de $35 \mathrm{~km} / \mathrm{h}$, ou seja, dentro de uma escala sinótica isso poderia ser resolvido com resoluções temporais de 3 e 6 horas.

$\mathrm{Na}$ análise dos parâmetros estatísticos para a altura significativa ficou claro que o modelo tende a subestimar os dados da boia e este padrão é observado em todos os casos (março, agosto e dezembro). A partir da análise por faixa de período, observa-se que essa subestimação não necessariamente ocorre para todos os sistemas do mar. Para as faixas entre 2 e 6 segundos, nos casos de março e de agosto, os resultados de Hs do modelo apresentaram valores superiores aos registrados pela boia. Para o caso de dezembro, no qual foi observado um predomínio maior de ondas entre 2 e $10 \mathrm{~s}$, os resultados do modelo subestimaram a boia. Esta análise por faixa de período evidenciou ainda que os erros para o parâmetro de Hs aumentam para ondas com períodos mais longos, registrando os maiores valores de EMQ para a faixa de períodos acima de 10 segundos. 
De uma maneira geral, as rodadas de $20 \mathrm{~km}, 60 \mathrm{~km}$ e $100 \mathrm{~km}$ apresentaram resultados semelhantes para os casos em que os sistemas meteorológicos associados à formação de ondas tinham dimensões espaciais grandes. Mas que para resoluções mais grosseiras, como a de 300 e $600 \mathrm{~km}$, os valores de viés e EMQ aumentaram consideravelmente.

No caso de dezembro ficou evidente o impacto que as diferenças entre as maiores e menores resoluções espaciais podem provocar. Isto ocorreu porque os sistemas meteorológicos responsáveis pelas ondas na Bacia de Campos em dezembro se formaram próximo à costa e tiveram dimensões espaciais singulares e menores que as grandes pistas observadas nos casos de março e agosto.

Desta forma, baseado nos resultados aqui apresentados, sugere-se como resolução ideal do campo de vento a de $60 \mathrm{~km}$, espera-se assim, representar melhor os eventos meteorológicos que geram pistas com dimensões espaciais menores e, consequentemente, minimizar os erros. As resoluções temporais apresentaram diferenças ínfimas entre si, o que indica que a resolução de 6 horas funciona satisfatoriamente. Mas ressalta-se que, para os casos de ciclones com velocidade de propagação muita alta, como por exemplo os ciclones explosivos, resoluções temporais mais refinadas como de 1 e $3 \mathrm{~h}$ poderão ser mais adequadas.

\section{AGRADECIMENTOS}

Os autores agradecem ao Laboratório de Instrumentação Oceanográfica - LIOC e ao Laboratório de Modelagem de Processos Marinhos e Atmosféricos - LAMMA pelo apoio disponibilizado.

\section{REFERÊNCIAS BIBLIOGRÁFICAS}

Cavaleri, L., Bertotti, L. 2003. The characteristics of wind and wave fields modelled with different resolutions. Quarterly Journal of the Royal Meteorological Society,129(590), pp.1647-1662.

Cavaleri, L., Bertotti, L. 2006. The Improvement of modelled wind and waves fields with increasing resolution. Ocean Engineering, 33(5), pp.553-565.

Freitas, S. R., Longo, K. M., Silva Dias, M. A. F., Chatfield, R., Silva Dias, P., Artaxo, P., Andreae, M. O., Grell, G., Rodrigues, L. F., Fazenda, A. and Panetta, J. 2009. The Coupled Aerosol and Tracer Transport model to the Brazilian developments on the Regional Atmospheric Modeling System (CATT-BRAMS) - Part 1: Model description and evaluation. Atmospheric Chemistry and Physics, 9 (8), pp. $2843-2861$.

Innocentini, V., Oliveira, F. A., Prado, S. C. S. C. 2003. Modelo de ondas aplicado ao caso 5-8 de maio de 2001. Revista Brasileira de Meteorologia, 18 (01), pp. 97-104.

Kalnay, E. 2003. Atmospheric, modeling, data assimilation and predictability. Cambridge University Press.

Mass, C. F., Ovens, D., Westrick, K. and Colle, B. A. 2002 Does Increasing Horizontal Resolution Produce More Skillful Forecasts? Bulletin of the American Meteorological Society, 83(3), pp.407430. 
Mendes, D. 2006. Regimes de circulação no Atlântico Sul e sua relação com a localização e intensidade de sistemas ativos e com o balanço de vapor na região. Tese de Doutorado. Universidade de Lisboa, Portugal.

Mendes, D., Moura, R. G. and Mendes, M. C. D. 2009. Estudo de caso de ciclone extratropical sobre a América do Sul: sensibilidade das análises. Revista Brasileira de Meteorologia, 24 (4) pp. 399-406.

Murray, R.J., Simmonds, I. 1991 A numerical scheme for tracking cyclone centres from digital data.Part I: development and operation of the scheme. Australian Meteorological Magazine, 39(3), pp.155-166.

Ostritz, F. F.; Violante-Carvalho, N.; Maturo, L. 2012. Estimativas de um modelo de geração e propagação de ondas empregando campos de vento com diferentes resoluções espaciais e temporais. Revista Brasileira de Meteorologia, 27(3), pp. 315-328.

Palmeira, A. C. P. de A.; Camargo, R de. and Palmeira, R. M. de J. 2012. Efeito do detalhamento dos ventos na modelagem de agitação marítima no oeste do Atlântico Sul. Revista Brasileira de Meteorologia. 27(1), pp. 117-125.

Parente, C. E. 1999. Uma Nova Técnica Espectral para Análise Direcional de Ondas. Tese de Doutorado, COPPE/Universidade Federal do Rio de Janeiro, Rio de Janeiro, Brasil.

Pesquero, J. F. 2001. Um Estudo de Avaliação Estatística do Modelo Global do CPTEC com Relação ao Número, Trajetória e Intensidade de Ciclones e Anticiclones para o Hemisfério Sul. Tese de Doutorado, INPE, São José dos Campos, Brasil.

Pinho, U. F. 2003. Caracterização dos Estados de Mar na Bacia de Campos. Dissertação de Mestrado. COPPE/Universidade Federal do Rio de Janeiro, Rio de Janeiro, Brasil.

Pielke, R. A., Cotton, W. R., Walko, R. L., Tremback, C. J., Lyons, W. A., Grasso, L. D., Nicholls, M. E., Moran, M. D., Wesley, D. A., Lee, T. J., and Copeland, J. H. 1992. A Comprehensive Meteorological Modeling System - RAMS. Meteorology and Atmospheric Physics, 49(1), pp. 6991.

Reboita, M. S. 2008. Ciclones Extratropicais sobre o Atlântico Sul: Simulação Climática e Experimentos de Sensibilidade. Tese de Doutorado, IAG-USP, São Paulo, SP, Brasil.

Rife, D. L., Davis, C. A. 2005. Verification of temporal variations in mesoscale numerical wind forecasts. Monthly Weather Review, 133(11), pp. 3368-3381.

Seixas, N. A. B. 1997. Clima de ondas na Bacia de Campos: Análise de dados e proposta de parametrização. Tese de Doutorado. Observatório Nacional, Rio de Janeiro, RJ, Brasil.

Silva, F.L.C. 2008. Investigações sobre a chegada de ondulações (swell) em situações de bom tempo na Bacia de Campos. Dissertação de Mestrado - COPPE/Universidade Federal do Rio de Janeiro, Rio de Janeiro, Brasil.

Tolman, H.L. 2009. User Manual and System Documentation of Wavewatch iii ${ }^{\mathrm{TM}}$ version 3.14, MMAB Technical Note, 276, pp. 220.

Tolman, H. L. and Chalikov. D. V. 1996. Source terms in a third-generation wind-wave model. Journal of Physical Oceanography, 26(11), pp. 2497-2518.

Tremback, C. J., Kessler, R. 1985. A surface temperature and moisture parameterization for use in mesoscale numerical models. Preprints. 7th AMS Conference on Numerical Weather Prediction. Montreal, Quebec, Canada, 17-20 June 1985, Amer. Meteor. Soc., Boston. 355-358. 
Tripoli, G. J., Cotton, W. R. 1982. The Colorado State University three-dimensional cloud mesoscale model, 1982: PartI: General theoretical framework and sensitivity experiments. J. de Rech. Atmos.. 16, pp. 185-220.

Vale, E. M. de J. P. 2012. Análise de extremos de onda de nordeste na Bacia de Campos. Dissertação de Mestrado. COPPE/Universidade Federal do Rio Janeiro, Rio de Janeiro, RJ, Brasil.

Violante-Carvalho, N. 1998. Investigação sobre a evolução do clima de ondas na Bacia de Campos e a correlação com as situações meteorológicas associadas. Dissertação de Mestrado, COPPE/ Universidade Federal do Rio Janeiro, Rio de Janeiro, Brasil.

Winter, W. R., Jahnert, R. J., França, A. B. 2007. Bacia de Campos. Boletim de Geociências da PETROBRAS. 15(2), pp. 511-529.

Zhong, S. and Fast, J. 2003. An evaluation of the MM5, RAMS, and Meso-Eta models at subkilometer resolution using VTMX field campaign data in the Salt Lake Valley. Monthly Weather Review,131(7), pp. 1301-1322.

Received in December 15, 2015.

Accepted in May 17, 2017. 